\section{The ch-TOG/XMAP215 protein is essential for spindle pole organization in human somatic cells}

\author{
Fanni Gergely, ${ }^{1}$ Viji M. Draviam, ${ }^{2,4}$ and \\ Jordan W. Raff ${ }^{3,5}$ \\ ${ }^{1}$ Department of Pharmacology, ${ }^{2}$ Department of Zoology, \\ and ${ }^{3}$ Department of Genetics, University of Cambridge, \\ Wellcome Trust/Cancer Research UK Institute, \\ Cambridge CB2 1QR, UK
}

The ch-TOG/XMAP215 family of proteins bind directly to microtubules and appear to play an essential role in stabilizing spindle microtubules. These proteins stabilize microtubules mainly by influencing microtubule plus-end dynamics, yet, in vivo, they are all strongly concentrated at spindle poles, where the minus ends of the microtubules are concentrated. In Drosophila embryos, the centrosomal protein D-TACC is required to efficiently recruit ch-TOG/Msps to centrosomes. In humans, ch-TOG and the three known TACC proteins have been implicated in cancer, but their functions are unknown. Here we extensively depleted TACC3 and chTOG from HeLa cells using RNA interference. In TACC3-depleted cells, spindles are well organized, but microtubules are partially destabilized and ch-TOG is no longer concentrated on spindle microtubules. In chTOG-depleted cells, relatively robust spindles form, but the spindles are highly disorganized. Thus, in human somatic cells, ch-TOG appears to play a major role in organizing spindle poles, and a more minor role in stabilizing spindle microtubules that is, at least in part, mediated via an interaction with TACC3.

Supplemental material is available at http://www.genesdev.org.

Received August 7, 2002; revised version accepted November 4, 2002.

The XMAP215/ch-TOG family of microtubule associated proteins (MAPs) are among the best studied microtubule-stabilizing proteins. In the presence of purified tubulin, these proteins stabilize microtubules mainly by stimulating growth rates at microtubule plus ends, whereas in Xenopus egg extracts they also increase microtubule plus-end stability by counteracting the activity of the microtubule catastrophe factor XKCM1 (Tournebize et al. 2000; Kinoshita et al. 2001). These proteins are essential for stabilizing spindle microtubules in both Xenopus egg extracts and worm embryos (Matthews et

[Keywords: ch-TOG; XMAP215; TACC; mitosis; mitotic spindle] ${ }^{4}$ Present address: Department of Biology, Massachusetts Institute of Technology, Cambridge, Massachusetts 02139, USA ${ }^{5}$ Corresponding author.

E-MAIL j.raff@welc.cam.ac.uk; FAX 44-1223-334089.

Article and publication are at http://www.genesdev.org/cgi/doi/10.1101/ $\operatorname{gad} .245603$. al. 1998; Tournebize et al. 2000; Popov et al. 2001). Surprisingly, although XMAP215/ch-TOG mainly influences microtubule plus-end dynamics, these proteins are strongly concentrated at centrosomes, where the minus ends of the microtubules are clustered (Nabeshima et al. 1995; Wang et al. 1997; Charrasse et al. 1998; Matthews et al. 1998; Cullen et al. 1999; Tournebize et al. 2000; Garcia et al. 2001).

In Drosophila, the centrosomal protein D-TACC has been shown to be required to efficiently recruit Msps/ ch-TOG to centrosomes (Cullen et al. 2001; Lee et al. 2001). The interaction between D-TACC and Msps/chTOG is highly conserved, and in human cells, all three known TACC proteins are concentrated at centrosomes and can interact with ch-TOG (Gergely et al. 2000; Cullen et al. 2001). Interestingly, ch-TOG and the three human TACC proteins have been implicated in cancer but the function of these proteins in mammalian cells is not known (Gergely 2002; Raff 2002). An attractive possibility is that these proteins function in human somatic cells as they do in frog and fly embryos, and are required to stabilize spindle microtubules. Perturbing their function could therefore lead to spindle defects and so contribute to the genetic instability that is essential for the development of cancer (Li et al. 1997; Brinkley and Goepfert 1998; Doxsey 1998; Salisbury et al. 1999).

\section{Results and Discussion}

To test whether TACC3 and ch-TOG play an essential role in cell division in humans, we used small interfering RNAs (siRNAs) to reduce the levels of each protein in HeLa cells (Elbashir et al. 2001). In cells transfected with TACC3 siRNA, the overall levels of TACC3 were reduced by $\sim 80 \%-90 \%$ after $48 \mathrm{~h}$ (Fig. $1 \mathrm{~A}$, lane 3 ). A similar treatment of cells with ch-TOG siRNA produced an $\sim 60 \%$ overall reduction in ch-TOG protein levels (Fig. 1, lane 3). Both proteins were also depleted to these respective levels in cells simultaneously treated with both siRNAs (Fig. 1B, lane 4). As the efficiency of transfection with siRNAs often varied from experiment to experiment and from cell to cell, we tested the levels of depletion in individual cells by immunofluorescence. We found that the levels of TACC3 and ch-TOG protein normally vary during the cell cycle, and are highest during mitosis (see Supplementary Fig. 1). We therefore only compared the levels of TACC3 and ch-TOG on centrosomes and spindles in mitotic cells. Approximately $70 \%$ of siRNA-treated mitotic cells had strongly reduced levels of TACC3 (scored as $<20 \%$ normal levels) or ch-TOG (scored as $<30 \%$ normal levels) on their centrosomes/ spindles, whereas the remaining $\sim 30 \%$ of mitotic cells had only weakly reduced or normal levels (Fig. 2).

In TACC3-depleted HeLa cells, the mitotic spindles were usually bipolar and well organized, and the microtubules did not appear to be dramatically destabilized (Figs. 2A, 3A). In $\sim 30 \%$ of all TACC3-depleted metaphase cells, however, at least one chromosome was not aligned properly at the metaphase plate (Fig. 3A,C, graph $5)$. There was a strong correlation between this chromosome alignment defect and the levels of TACC3 observed in a cell by immunofluorescence. Cells with high levels of TACC3 rarely had a chromosome alignment defect $(<5 \%)$, whereas most $(-70 \%)$ cells that had no, or 


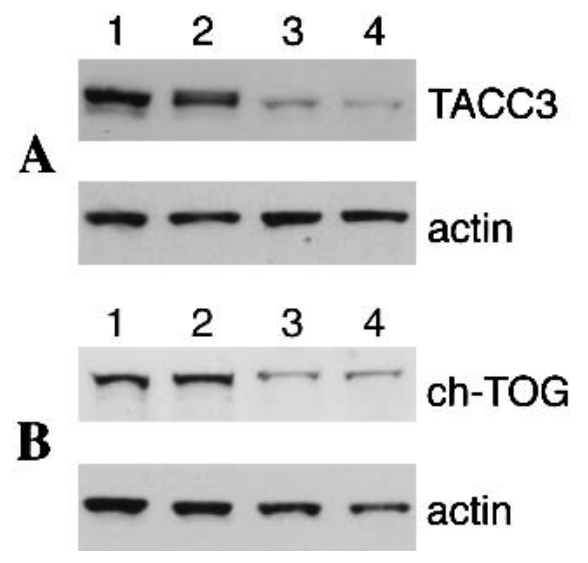

Figure 1. TACC 3 and ch-TOG protein levels are reduced by siRNA treatment. A Western blot showing the levels of TACC3 $(A)$ and ch-TOG $(B)$ after $48 \mathrm{~h}$ of siRNA treatment. Actin is shown as a loading control. (A) Mock-depleted cells (lane 1), ch-TOG-depleted cells (lane 2), TACC3-depleted cells (lane 3), and TACC3- and ch-TOG-depleted cells (lane 4). (B) Mock-depleted cells (lane 1), TACC3-depleted cells (lane 2), ch-TOGdepleted cells (lane 3), and ch-TOG- and TACC3-depleted cells (lane 4).

very little, detectable TACC3 showed the chromosome alignment defect. In addition, TACC3-depleted cells had an approximately threefold-higher mitotic index than control cells (Fig. 3C, graph 1), suggesting that the failure to properly align chromosomes at metaphase was triggering the spindle assembly checkpoint (Shah and Cleveland 2000). This delay in mitosis appeared to be transient, however, as a small number of TACC3-depleted cells were observed exiting mitosis relatively normally even when no TACC3 was detectable on the spindle (data not shown).

In ch-TOG-depleted mitotic cells, the spindle microtubules did not appear to be dramatically destabilized, but the spindles were usually highly disorganized (Figs. 2B, 3B,C, graph 6). As in TACC3-depleted cells, chromosomes often failed to align properly at the metaphase plate, but this defect was much stronger in ch-TOG-depleted cells (Fig. 3B,C, graph 3), and the mitotic index in these cells was elevated by approximately fivefold (Fig. 3C, graph 1). Even these highly disorganized cells, however, were capable of eventually exiting mitosis, and we observed many cells in interphase with an aberrant DNA content (Fig. 3C, graph 2). In both TACC3- and ch-TOGdepleted cells, we saw no obvious defects in microtubule organization in interphase cells.

These observations demonstrate that both TACC 3 and ch-TOG are essential for mitosis in HeLa cells. Surprisingly, however, microtubules did not appear to be dramatically destabilized by the depletion of either protein. To test whether spindle microtubules were subtly destabilized by the depletion of TACC 3 or ch-TOG, we attempted to quantify the intensity of spindle fluorescence in control and depleted cells. As fluorescence levels often varied between different coverslips, we selected areas of siRNA-treated coverslips that contained at least one strongly depleted cell and a cell that had normal levels of TACC3 or ch-TOG. We then optically sectioned each cell and calculated the average pixel intensity in a manually defined area that contained the entire spindle region
(Fig. 4; see Materials and Methods). This comparison proved relatively straightforward for TACC3-depleted cells, as the spindles in these cells were usually well organized and bipolar. The microtubule density in these spindles was reduced by $\sim 20 \%$ (Fig. 4A). In ch-TOG-depleted cells, however, the spindles were so disorganized that it was impossible to objectively define the spindle area, and no meaningful quantitative comparison could be made.

In an attempt to overcome this problem, we treated cells with siRNAs for $24 \mathrm{~h}$ and then chilled them for $2 \mathrm{~h}$ to depolymerize the microtubules. We then warmed the cells to allow the microtubules to regrow for varying lengths of time before fixing the cells for analysis. Again, we analyzed areas of an siRNA-treated coverslip that contained at least one strongly depleted cell and a cell that had normal levels of TACC3 or ch-TOG. This assay produced a striking qualitative difference between the rates of microtubule regrowth in depleted and nondepleted cells. After 25 min of recovery, relatively normal bipolar spindles with the majority of chromosomes aligned at a metaphase plate had reformed in the majority of mitotic cells $(\sim 60 \%)$ that were not depleted of either protein (Fig. 4B,C). In TACC3-depleted cells, the spindles that reformed were usually bipolar and well or-
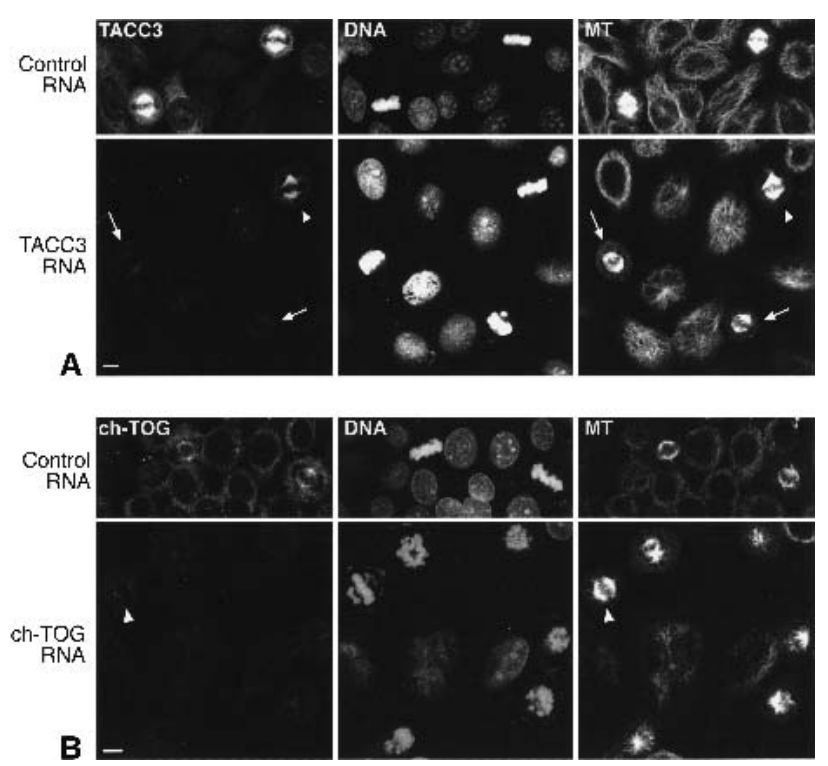

Figure 2. Immunofluorescence analysis of TACC3 and chTOG protein levels in siRNA-treated cells. (A) Mock-depleted (top panels) and TACC3-depleted (bottom panels) HeLa cells stained to reveal the distribution of TACC3, DNA, and microtubules. In this and all subsequent experiments, images were acquired from experimental and control cells that were treated and processed for immunofluorescence at the same time using identical settings on the confocal microscope. The TACC3 siRNA treatment has strongly reduced TACC3 levels in two mitotic cells (arrows), but substantial levels of TACC3 remain in one mitotic cell (arrowhead). (B) Mock-depleted (top panels) and ch-TOG-depleted (bottom panels) HeLa cells stained to reveal the distribution of ch-TOG, DNA, and microtubules. The ch-TOG siRNA treatment strongly reduced ch-TOG levels in most cells visible in this field, but small amounts of protein are still detectable on the centrosomes and spindles of some mitotic cells (arrowhead). Bar, $10 \mu \mathrm{m}$. 
A
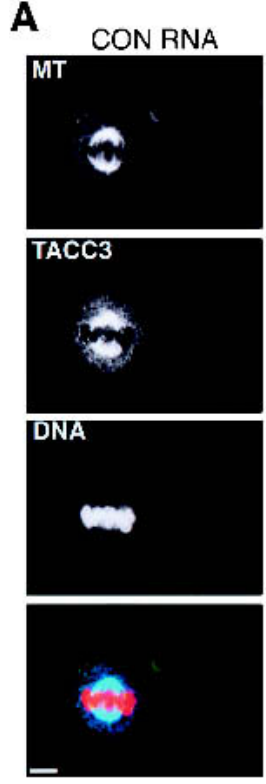

c
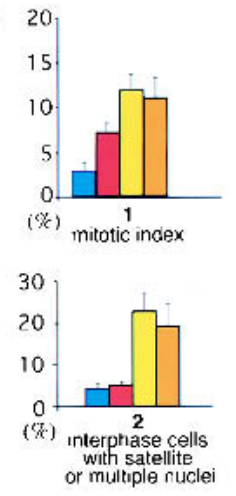
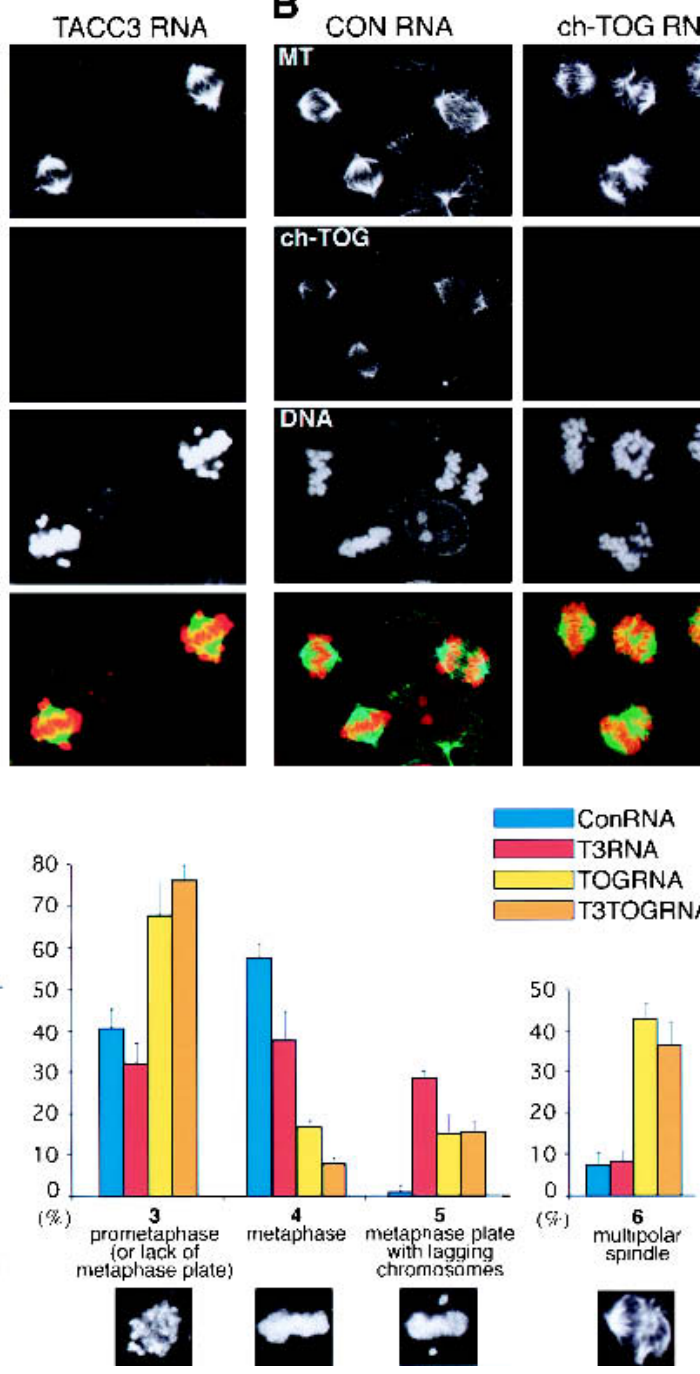

Figure 3. Mitotic defects in TACC3- and ch-TOG-depleted HeLa cells. $(A, B)$ Mock-depleted (left panels) and either TACC3-depleted ( $A$, right panels) or chTOG-depleted (B, right panels) cells were stained to reveal the distribution of microtubules (green in merged image), TACC3 or ch-TOG (blue in merged image), and DNA (red in merged image). Bar, $10 \mu \mathrm{m}$. $(C)$ Quantitation of the defects observed in TACC3- and ch-TOG-depleted cells. In all graphs, the percentage of cells exhibiting a particular phenotype is indicated for mock-depleted (blue bars), TACC3-depleted (red bars), ch-TOG-depleted (yellow bars), or TACC3- and chTOG-depleted (orange bars) cells. (Graph 1) The mitotic index in the population of cells as determined by phospho-histone H3 staining. (Graph 2) The percentage of interphase cells exhibiting multiple or satellite nuclei. (Graph 3) The percentage of mitotic cells in a prometaphase-like state (with no discernible metaphase plate). (Graph 4) The percentage of mitotic cells with a normal metaphase plate. (Graph 5) The percentage of mitotic cells with a clear metaphase plate, but with at least one chromosome not aligned at the equator. (Graph 6) The percentage of mitotic cells with more than two spindle poles. Two-hundred-fifty cells were scored from each of four independent experiments for both the mitotic index and the multinucleated phenotype. Fifty metaphase cells from two independent experiments were scored for the prometaphase, metaphase, and lagging chromosome phenotypes. Thirty mitotic cells were scored from each of four independent experiments for the presence of multipolar spindles. Error bars represent the standard deviation.

ganized, but they had an $\sim 30 \%$ lower density of microtubules (Fig. 4A), and only $\sim 25 \%$ had successfully aligned the majority of their chromosomes at a metaphase plate (Fig. 4B). In ch-TOGdepleted cells, only $\sim 10 \%$ of mitotic cells had successfully aligned the majority of their chromosomes on a metaphase plate (Fig. 4B), and microtubule density was clearly qualitatively lower than normal (Fig. 4C). Again, however, this difference in microtubule density was impossible to quantify, as these spindles were too disorganized (Fig. 4C). These observations strongly suggest that microtubules are partially destabilized by the depletion of TACC3 and ch-TOG.

It was previously shown that D-TACC is required to efficiently recruit ch-TOG/ Msps to centrosomes and spindle poles in Drosophila embryos and oocytes (Cullen et al. 2001; Lee et al. 2001). In TACC3-depleted cells, however, the level of ch-TOG at centrosomes was only slightly, if at all, reduced (Fig. 5A). This may be because ch-TOG can still be recruited to spindle poles by TACC 1 or TACC2, which remain concentrated at centrosomes in TACC3depleted cells (data not shown). Unexpectedly, however, the level of ch-TOG on the spindle was greatly reduced in TACC3depleted cells (Fig. 5A). This result is very surprising, as ch-TOG can bind directly to purified microtubules in vitro (Spittle et al. 2000). In human somatic cells, it seems that ch-TOG requires TACC3 to bind efficiently to spindle microtubules. This failure to recruit ch-TOG to mitotic spindles presumably explains why spindle microtubules are partially destabilized in TACC3depleted cells.

In contrast, the depletion of ch-TOG did not appear to disrupt the localization of TACC3 (Fig. 5B), TACC1, or TACC2 (data not shown) on centrosomes or spindles. As the depletion of ch-TOG was never as efficient as the depletion of TACC3 (Fig. 1), it is possible that the residual ch-TOG is required to efficiently localize the TACC proteins to centrosomes/spindles.

The depletion of either TACC3 or chTOG did not seem to affect the centrosomal localization of $\gamma$-tubulin (see Supplementary Fig. 2), TACC1, or TACC2 (data not shown), and all of these proteins were recruited to the extra spindle poles often found in the ch-TOG-depleted cells. This raised the possibility that these disorganized spindle poles might not arise from a defect in spindle pole organization, but rather from the presence of extra centrosomes accumulated from previously aberrant rounds of mitosis. To test this possibility, we followed mitosis in living HeLa cells during their first round of division after ch-TOG depletion (see Supplementary Fig. 3). Initially, we attempted to do this by depleting ch-TOG in synchronized cells that were injected with a GFP-tubulin construct, allowing us to fol- 

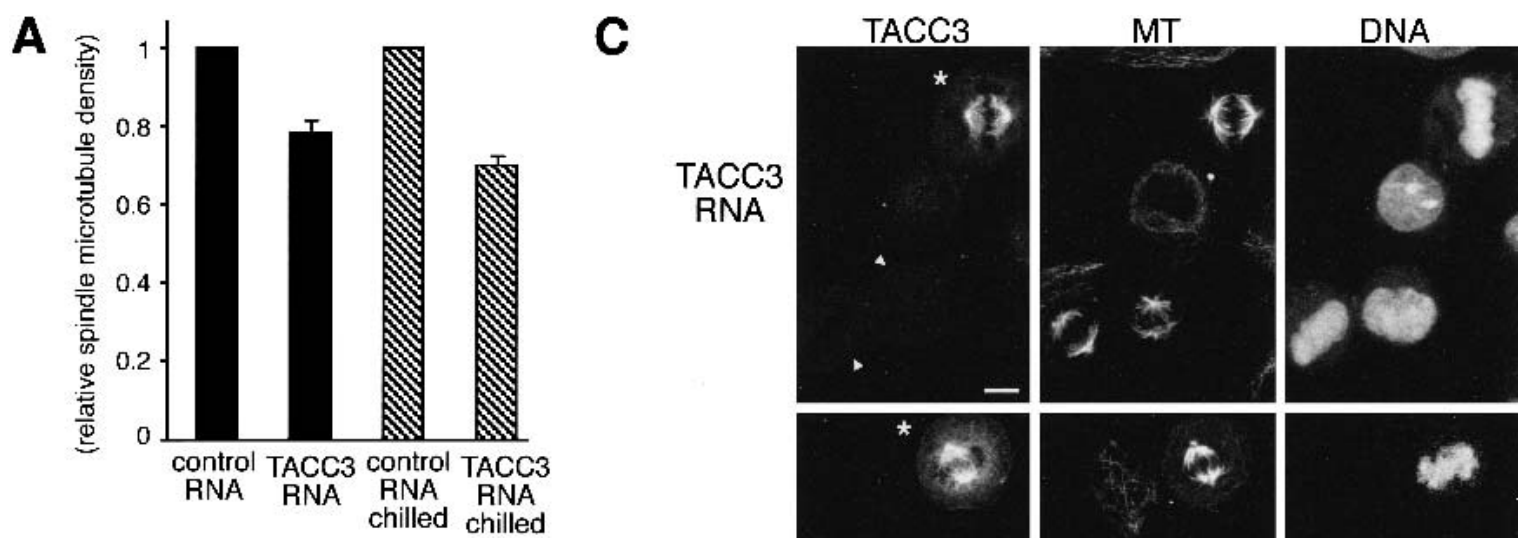

B
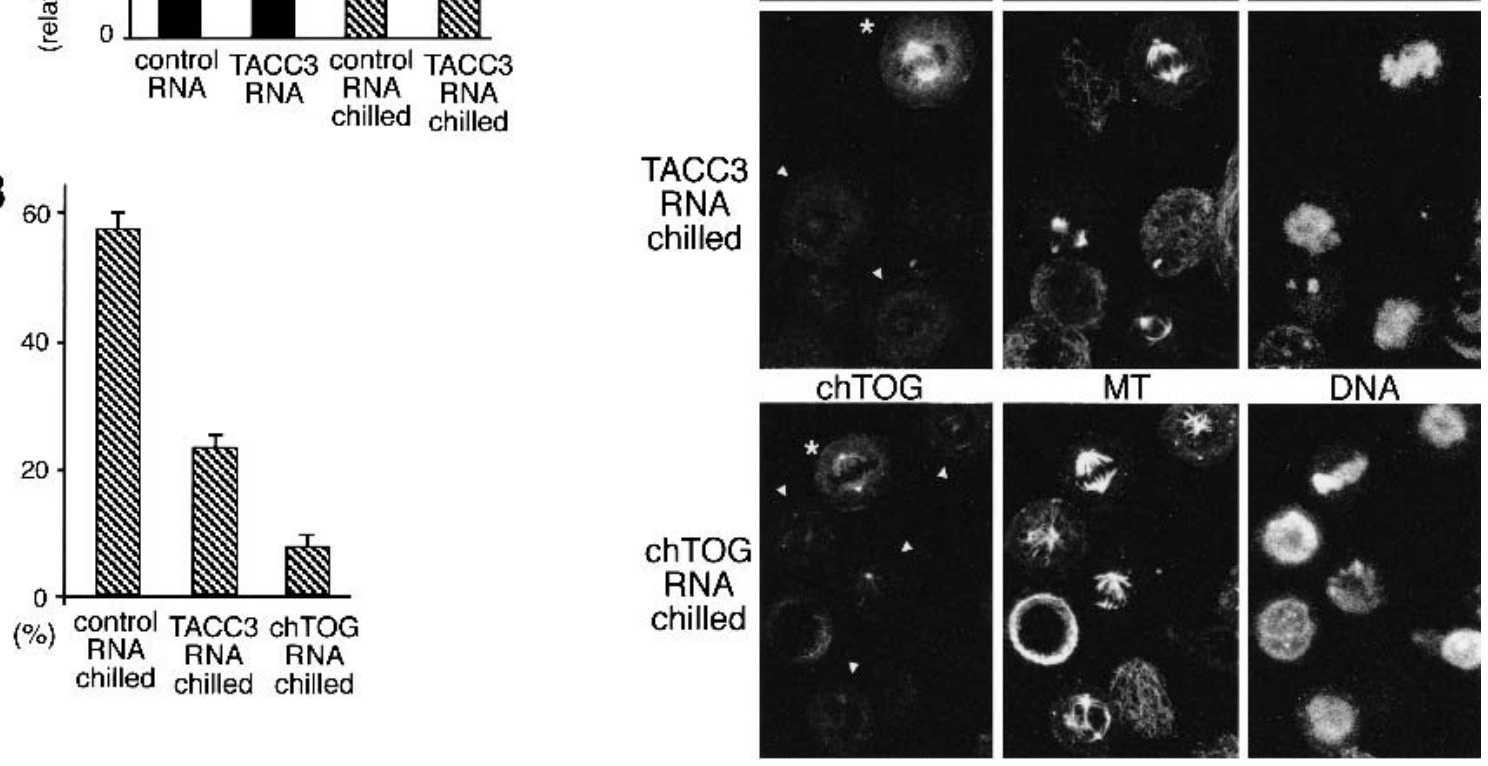

Figure 4. The depletion of TACC 3 and ch-TOG partially destabilizes spindle microtubules. (A) A graph showing the relative spindle fluorescence intensity in nondepleted or TACC3-depleted mitotic cells (black bars), or in nondepleted or TACC3-depleted cells that have been chilled and allowed to recover for $25 \mathrm{~min}$ (hatched bars). Note that the spindle fluorescence of both sets of nondepleted cells has been normalized to 1. (B) A graph showing the percentage of cells that have properly aligned the majority of their chromosomes at a metaphase plate after $25 \mathrm{~min}$ of recovery from cold treatment. Error bars represent the standard deviation. $(C$, top panels) A field of cells treated with TACC3 siRNA. One mitotic cell (marked with ${ }^{\star}$ ) has not been significantly depleted of TACC3, but two other mitotic cells (marked with arrowheads) have been significantly depleted. Fields such as these were used to compare the spindle microtubule density in the depleted and nondepleted cells (see Materials and Methods). Fields of TACC3 (middle panels) and ch-TOG (bottom panels) siRNA-treated cells that were chilled to depolymerize the microtubules and then allowed to recover for 25 min. In each panel, a nondepleted mitotic cell is marked with ${ }^{\star}$, and depleted cells are marked with arrowheads. Bar, $10 \mu \mathrm{m}$.

low the behavior of microtubules. Unfortunately, GFPtubulin proved to be deleterious to cells, and very few injected cells entered mitosis. We therefore followed the behavior of spindle poles indirectly, by monitoring the behavior of ch-TOG-depleted cells that were coinjected with a histone-GFP construct. All $10 \mathrm{ch}$-TOG-depleted cells observed in this way entered a prolonged prometaphase period in which some of the chromosomes repeatedly aligned on a metaphase plate-like structure (that often appeared to be organized by multiple poles), and then reverted to a more disorganized state (Supplementary Fig. 3). Eight of these cells eventually exited mitosis, and the chromosomes were often segregated unevenly between multiple poles, although chromosome segregation and cytokinesis appeared to occur at normal rates. None of these defects were observed in mock-injected control cells. Thus, spindle pole organization is disrupted even during the first round of mitosis after chTOG depletion.

Our results have two important implications for mechanisms of TACC 3 and ch-TOG function in human cells. First, TACC3 is essential for mitosis in HeLa cells.

This result is important because a recent study reported that TACC3 knockout mice die during embryogenesis due to a massive increase in apoptosis, and this phenotype is partially rescued by lowering p53 levels (Piekorz et al. 2002). Surprisingly, however, TACC3-/ -;p53-/- cells have only a slight increase in chromosomal abnormalities, suggesting that TACC3 is not essential for spindle function in mice. We suspect that TACC3 knockout mice, like TACC3-depleted human cells, have high levels of relatively subtle mitotic defects that cause a transient arrest in mitosis. These transiently arrested cells would then normally be eliminated by apoptosis in a p53-dependent manner during the next cell cycle, even if no gross chromosomal abnormalities were generated (Lanni and Jacks 1998; Ciciarello et al. 2001).

Second, although ch-TOG/XMAP215 plays an essential role in stabilizing spindle microtubules in frog egg extracts and worm embryos (Matthews et al. 1998; Tour- 

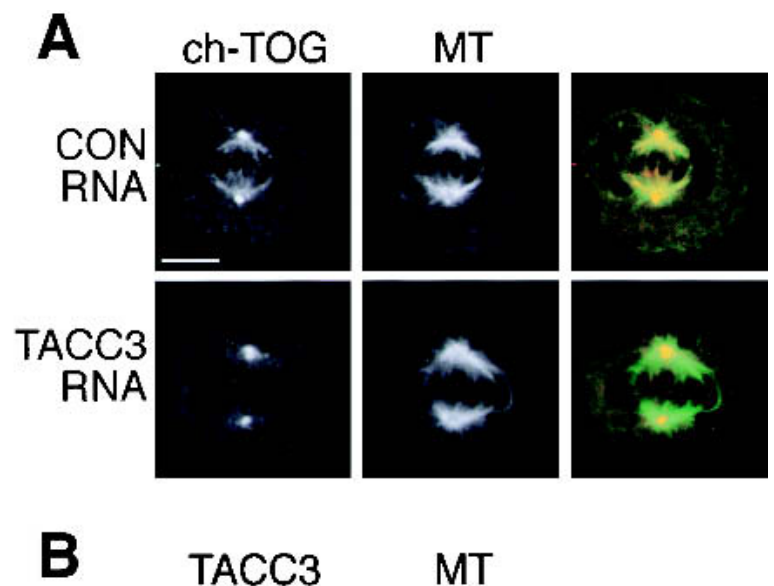

TACC3
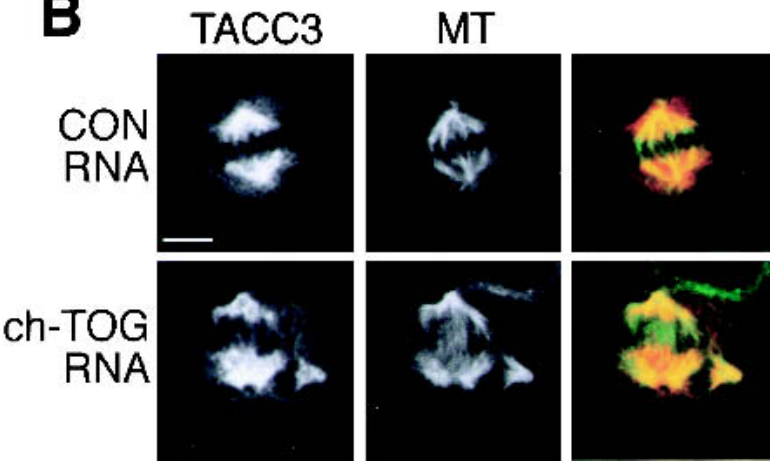

Figure 5. The localization of ch-TOG is disrupted in TACC3depleted cells. (A) The distribution of ch-TOG (left panels, red in merged image) and microtubules (middle panels, green in merged image) in mock-depleted (top panels) and TACC3-depleted (bottom panels) cells. Ch-TOG is detectable on centrosomes, but is not detectable on spindle microtubules in the TACC3-depleted cells. (B) The distribution of TACC3 (left panels, red in merged image) and microtubules (middle panels, green in merged image) in mock-depleted (top panels) and chTOG-depleted (bottom panels) cells. TACC3 remains concentrated on spindles in the ch-TOG-depleted cells. Bar, $10 \mu \mathrm{m}$.

nebize et al. 2000; Popov et al. 2001), in human somatic cells it appears to play a more minor role in stabilizing spindle microtubules, and a more major role in organizing spindle poles. This may reflect a difference between embryonic and somatic cell types, as the phenotype in ch-TOG-depleted HeLa cells is reminiscent of that observed in Drosophila msps mutant larval brain cells that are partially depleted of Msps/ch-TOG /Cullen et al. 1999). In frog egg extracts, XMAP215 stabilizes microtubules primarily by counteracting the microtubule-destabilizing activity of XKCM1/Kin1 (Desai et al. 1999). Perhaps XKCM1 is less active in somatic cells, so it is less important for XMAP215/ch-TOG to counteract its activity. It was recently shown that the changes in microtubule dynamics that occur during the cell cycle are different in mammalian tissue culture cells and Xenopus egg extracts (Rusan et al. 2001).

How might ch-TOG function to organize spindle poles in somatic cells? When overexpressed in HeLa cells, the TACC proteins form large polymers in the cytoplasm that also contain ch-TOG (Gergely et al. 2000). Perhaps the TACCs and ch-TOG can form a structural lattice at centrosomes that functions both to maintain the integrity of spindle poles and to stabilize spindle microtu- bules. In the TACC3-depleted cells, the spindle pole organizing function might not be disrupted because TACC1 and/or TACC2 can still recruit ch-TOG to centrosomes. We are currently attempting to test this possibility by simultaneously depleting all three TACC proteins from HeLa cells. Regardless of its mechanism, the role of ch-TOG/XMAP215 in organizing spindle poles may explain the longstanding mystery of why this potent regulator of microtubule plus-end dynamics is so strongly concentrated at centrosomes.

\section{Materials and methods}

\section{Immunofluoresence}

Human HeLa cells were cultured, fixed, and stained with antibodies as described (Gergely et al. 2000). Rabbit anti-ch-TOG antibodies (Dionne et al. 2000; Tournebize et al. 2000), and rabbit anti-TACC3 antibodies (Gergely et al. 2000) were described previously. DM1 anti- $\alpha$-tubulin antibody (Sigma) was used at 1/1000 dilution, and GTU-88 anti- $\gamma$-tubulin antibody (Sigma) was used at 1/500 dilution. Phospho-histone H3 antibody (Upstate Biotechnology) was used at 1/1000 dilution to identify mitotic cells. Appropriate Alexa488- (Molecular Probes), Cy5-, or Cy3 (Jackson)-coupled secondary antibodies were used at 1/500 dilution. DNA was detected with either propidium iodide or TOTO. All imaging was performed using a Bio-Rad Radiance 2000 scanning confocal microscope using identical settings on the confocal microscope. The images were imported into Adobe Photoshop 6.0, and all of the images in any individual figure were processed in exactly the same way.

\section{RNA preparation and transfection}

Twenty-one-nucleotide (nt) RNAs were synthesized, deprotected, and gel-purified by Dharmacon. RNAs were annealed as described (Elbashir et al. 2001). The siRNA sequences targeting ch-TOG corresponded to region 5928-5948 nt, and the siRNA targeting TACC3 corresponded to region 83-103 nt of the coding sequence. The control RNA duplex used in all mock siRNA experiments corresponded to a 21-nt-long region of Fanconi anemia protein E. Cells were seeded on coverslips to $60 \%$ confluence in 24 -well plates, $16 \mathrm{~h}$ before transfection. We transfected 80 pmole of RNA duplex per well using the Oligofectamine reagent (Life Technologies) according to the manufacturer's instructions.

\section{Western blotting}

Whole-cell extracts were made by pelleting HeLa cells and boiling the pellet in SDS sample buffer. The extracts were separated by SDS-PAGE and blotted to nitrocellulose. Blots were incubated with primary antibodies at $1 \mu \mathrm{g} / \mathrm{mL}$ final concentration, and antibody binding was detected using the Supersignal kit (Pierce) according to the manufacturer's instructions. For quantitation, the blots were scanned into Adobe Photoshop and then imported into NIH Image 1.62. The total pixel intensity of each band was counted, and the degree of depletion was calculated after normalizing this figure to the total pixel intensity of an actin control.

Microtubule regrowth assay and spindle intensity quantification Twenty-four hours after the addition of the siRNA/oligofectamine solution, cells were transferred to $4^{\circ} \mathrm{C}$ for $2 \mathrm{~h}$. Cells were then recovered at $37^{\circ} \mathrm{C}$ and fixed at various time points. Cells were stained with anti- $\alpha-$ tubulin and anti-TACC3 or ch-TOG antibodies. Individual mitotic cells were optically sectioned using a confocal laser scanning microscope. These 3D datasets (z-series of 14-en face optical sections, 1-um steps) were projected onto a single image plane. Each channel of the projected image was inverted in NIH Image 1.62. We measured spindle microtubule intensity by defining the spindle area manually (containing the centrosomes and all visible microtubule bundles projecting towards chromosomes, but excluding astral microtubules), and calculated the mean pixel intensity per unit area of spindle. The same approach was applied to measure TACC3 antibody staining intensity in the spindle area. Having obtained both measurements for each cell, we scored them according to their TACC3 levels and divided them into two groups. The first group consisted of images of cells from the TACC3 RNA-treated coverslips that exhibited comparable levels (within 10\%) of TACC3 in untreated cells, and cells in the second group had at least a threefold reduction in TACC3 
levels. Each bar in Figure 4A represents data collected from three independent experiments, in which 20 cells $(10$ control and 10 TACC3-depleted) were scored. Datasets were normalized to control within each experiment.

\section{Acknowledgments}

We thank Duane Compton, Tony Hyman, K.J. Patel, and G. Wahl for reagents. We thank Jon Pines and members of the Raff lab for comments on the manuscript. This work was supported by a Royal Society Dorothy Hodgkin Fellowship (F.G.), a Cambridge Nehru Scholarship (V.M.D.), and a Wellcome Trust Senior Research Fellowship (J.R.)

The publication costs of this article were defrayed in part by payment of page charges. This article must therefore be hereby marked "advertisement" in accordance with 18 USC section 1734 solely to indicate this fact.

\section{References}

Brinkley, B.R. and Goepfert, T.M. 1998. Supernumerary centrosomes and cancer: Boveri's hypothesis resurrected. Cell Motil. Cytoskeleton 41: $281-288$

Charrasse, S., Schroeder, M., Gauthier-Rouviere, C., Ango, F., Cassimeris, L., Gard, D.L., and Larroque, C. 1998. The TOGp protein is a new human microtubule-associated protein homologous to the Xenopus XMAP215. J. Cell Sci. 111: 1371-1383.

Ciciarello, M., Mangiacasale, R., Casenghi, M., Zaira Limongi, M., D'Angelo, M. Soddu., S., Lavia, P., and Cundari, E. 2001. p53 displacement from centrosomes and p53-mediated G1 arrest following transient inhibition of the mitotic spindle. J. Biol. Chem. 276: 1920519213.

Cullen, C.F. and Ohkura, H. 2001. Msps protein is localized to acentrosomal poles to ensure bipolarity of Drosophila meiotic spindles. Nat. Cell Biol. 3: 637-642.

Cullen, C.F., Deak, P., Glover, D.M., and Ohkura, H. 1999. Mini spindles. A gene encoding a conserved microtubule-associated protein required for the integrity of the mitotic spindle in Drosophila. J. Cell Biol. 146: 1005-1018.

Desai, A., Verma, S., Mitchison, T.J., and Walczak, C.E. 1999. Kin I kinesins are microtubule-destabilizing enzymes. Cell 96: 69-78.

Dionne, M.A., Sanchez, A., and Compton, D.A. 2000. ch-TOGp is required for microtubule aster formation in a mammalian mitotic extract. J. Biol. Chem. 275: 12346-12352.

Doxsey, S. 1998. The centrosome-A tiny organelle with big potential. Nat. Genet. 20: 104-106.

Elbashir, S.M., Harborth, J., Lendeckel, W., Yalcin, A., Weber, K., and Tuschl, T. 2001. Duplexes of 21-nucleotide RNAs mediate RNA interference in cultured mammalian cells. Nature 411: 494-498.

Garcia, M.A., Vardy, L., Koonrugsa, N., and Toda, T. 2001. Fission yeast ch-TOG/XMAP215 homologue Alp14 connects mitotic spindles with the kinetochore and is a component of the Mad2-dependent spindle checkpoint. EMBO J. 20: 3389-3401.

Gergely, F. 2002. Centrosomal TACCtics. Bioessays 10: 915-925.

Gergely, F., Karlsson, C., Still, I., Cowell, J., Kilmartin, J., and Raff, J.W. 2000. The TACC domain identifies a new family of proteins that can interact with centrosomes and microtubules. Proc. Natl. Acad. Sci. 97: 14352-14357

Kinoshita, K., Arnal, I., Desai, A., Drechsel, D.N., and Hyman, A.A. 2001 Reconstitution of physiological microtubule dynamics using purified components. Science 294: 1340-1343.

Lanni, J.S. and Jacks, T. 1998. Characterization of the p53-dependent postmitotic checkpoint following spindle disruption. Mol. Cell Biol. 18: 1055-1064.

Lee, M.J., Gergely, F., Jeffers, K., Peak-Chew, S.Y., and Raff, J.W. 2001. Msps/XMAP215 interacts with the centrosomal protein D-TACC to regulate microtubule behaviour. Nat. Cell Biol. 3: 643-649.

Li, R., Yerganian, G., Duesberg, P., Kraemer, A., Willer, A., Rausch, C., Hehlmann, R. 1997. Aneuploidy correlated $100 \%$ with chemical transformation of Chinese hamster cells. Proc. Natl. Acad. Sci. 94: $14506-14511$.

Matthews, L.R., Carter, P., Thierry-Mieg, D., and Kemphues, K. 1998. ZYG-9, a Caenorhabditis elegans protein required for microtubule organization and function, is a component of meiotic and mitotic spindle poles. J. Cell Biol.. 141: 1159-1168.

Nabeshima, K., Kurooka, H., Takeuchi, M., Kinoshita, K., Nakaseko, Y., and Yanagida, M. 1995. p93dis1, which is required for sister chromatid separation, is a novel microtubule and spindle pole body-associating protein phosphorylated at the Cdc2 target sites. Genes and Dev 9: $1572-1585$

Piekorz, R.P., Hoffmeyer, A., Duntsch, C.D., McKay, C., Nakajima, H., Sexl, V., Snyder, L., Rehg, J., and Ihle, J.N. 2002. The centrosomal protein TACC3 is essential for hematopoietic stem cell function and genetically interfaces with p53-regulated apoptosis. $Е M B O ~ J .21: 653-$ 664.

Popov, A.V., Pozniakovsky, A., Arnal, I., Antony, C., Ashford, A.J., Kinoshita, K., Tournebize, R., Hyman, A.A., and Karsenti, E. 2001. XMAP215 regulates microtubule dynamics through two distinct domains. EMBO J. 20: 397-410.

Raff, J.W. 2002. Centrosomes and cancer: Lessons from a TACC. Trends Cell Biol. 12: 222-225.

Rusan, N.M., Fagerstrom, C.J., Yvon, A.M., and Wadsworth, P. 2001. Cell cycle-dependent changes in microtubule dynamics in living cells expressing green fluorescent protein- $\alpha$ tubulin. Mol. Biol. Cell 12: 971980.

Salisbury, J.L., Whitehead, C.M., Lingle, W.L., and Barrett, S.L. 1999. Centrosomes and cancer. Biol. Cell 91: 451-460.

Shah, J.V. and Cleveland, D.W. 2000. Waiting for anaphase: Mad2 and the spindle assembly checkpoint. Cell 103: 997-1000.

Spittle, C., Charrasse, S., Larroque, C., and Cassimeris, L. 2000. The interaction of TOGp with microtubules and tubulin. I. Biol. Chem. 275: 20748-20753.

Tournebize, R., Popov, A., Kinoshita, K., Ashford, A.J., Rybina, S., Pozniakovsky, A., Mayer, T.U., Walczak, C.E., Karsenti, E., and Hyman, A.A. 2000. Control of microtubule dynamics by the antagonistic activities of XMAP215 and XKCM1 in Xenopus egg extracts. Nat. Cell Biol. 2: 13-19.

Towbin, H., Staehlin, T., and Gordon, J. 1979. Electrophoretic transfer of proteins from polyacrylamide gels to nitrocellulose sheets: Procedures and some applications. Proc. Natl. Acad. Sci. 80: 4350-4354.

Wang, P.J. and Huffaker, T.C. 1997. Stu2p: A microtubule-binding protein that is an essential component of the yeast spindle pole body. J. Cell Biol. 139: 1271-1280. 


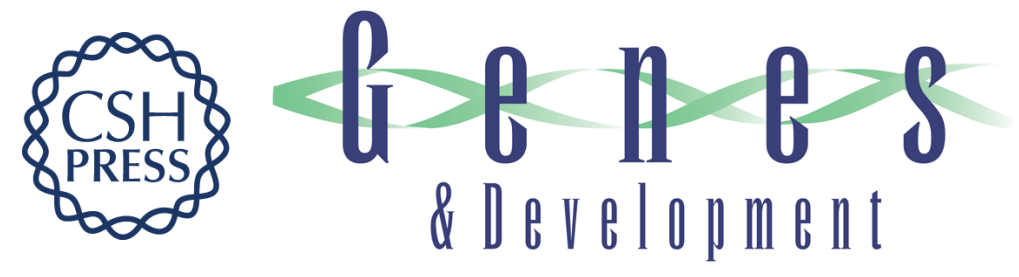

\section{The ch-TOG/XMAP215 protein is essential for spindle pole organization in human somatic cells}

Fanni Gergely, Viji M. Draviam and Jordan W. Raff

Genes Dev. 2003, 17:

Access the most recent version at doi:10.1101/gad.245603

Supplemental http://genesdev.cshlp.org/content/suppl/2003/02/03/17.3.336.DC1
Material

References This article cites 26 articles, 15 of which can be accessed free at: http://genesdev.cshlp.org/content/17/3/336.full.html\#ref-list-1

License

Email Alerting

Receive free email alerts when new articles cite this article - sign up in the box at the top Service right corner of the article or click here.

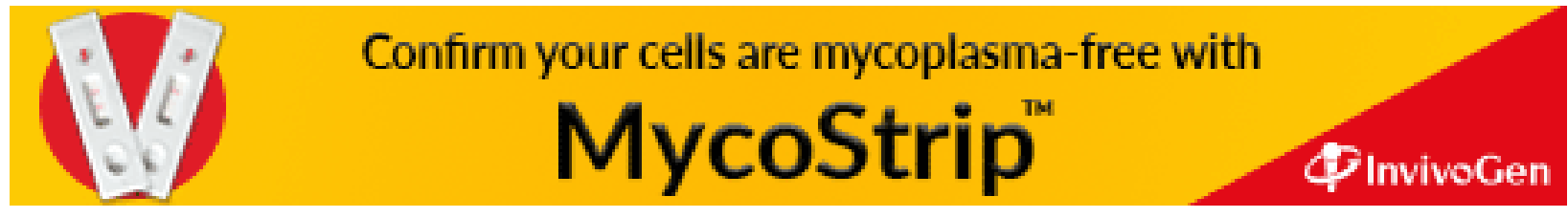

